\title{
Tumor necrosis factor $\alpha$-induced protein 8-like 1 promotes apoptosis by regulating $B$-cell leukemia/lymphoma-2 family proteins in RAW264.7 cells
}

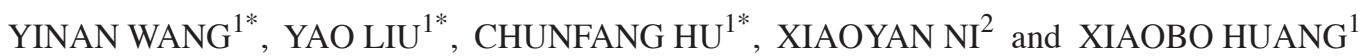 \\ ${ }^{1}$ Department of Immunology, Basic Medicine Science, Medical College, Xiamen University, Xiamen, Fujian 361005; \\ ${ }^{2}$ Shaoxing People's Hospital, Shaoxing Hospital of Zhejiang University, Shaoxing, Zhejiang 312000, P.R. China
}

Received May 26, 2015; Accepted August 19, 2016

DOI: $10.3892 / 01.2016 .5090$

\begin{abstract}
Although the newly identified protein tumor necrosis factor $\alpha$-induced protein 8 -like 1 (TNFAIP8L1), also known as TIPE1, has been reported to be able to induce apoptosis in human hepatocellular carcinoma cells, the involvement of TIPE1 in apoptosis remains to be elucidated. The present study investigated the pro-apoptotic effect of TIPE1 in an murine macrophage cell line, RAW264.7. The cell apoptosis rate was detected by flow cytometry. The results revealed that overexpressed TIPE1 could directly enhance the apoptosis and the cisplatin-induced cell death of RAW264.7 cells in vitro. Meanwhile, TIPE1 overexpression could suppress tumor growth in vivo. Furthermore, western blotting revealed that overexpressed TIPE1 could upregulate the expression of B-cell leukemia/lymphoma (Bcl)-2 associated X protein (Bax), Bcl-2 interacting killer (Bik) and p53 upregulated modulator of apoptosis (Puma), and activate the mitogen activated protein kinases (MAPKs) signaling pathway. However, western blotting demonstrated that inhibitors of the MAPKs pathway could not decrease the expression of Bax, Bik or Puma. These results indicated that TIPE1 could promote the apoptosis of RAW264.7 cells by upregulating the pro-apoptotic members of the Bcl-2 family of proteins, and that the MAPKs signaling pathway was not involved in the pro-apoptotic effect of TIPE1.
\end{abstract}

Correspondence to: Dr Yinan Wang, Department of Immunology, Basic Medicine Science, Medical College, Xiamen University, 422 Siming Nan Road, Xiamen, Fujian 361005, P.R. China

E-mail: yinanwang@xmu.edu.cn

*Contributed equally

Key words: tumor necrosis factor- $\alpha$-induced protein 8-like 1, apoptosis, B-cell leukemia/lymphoma-2 family, mitogen activated protein kinases, RAW264.7 cells

\section{Introduction}

It is generally accepted that certain death effector domain (DED)-containing proteins are involved in the progression of apoptosis and cell activation (1). Due to their similar protein structures, Sun et al (2) identified four members of the tumor necrosis factor $\alpha$-induced protein- 8 (TNFAIP8, also known as TIPE) family, named TIPE, TIPE1, TIPE2 and TIPE3. There are at least two members of the protein family that have been identified to have a DED-like domain $(2,3)$. However, these two proteins separately presented opposite functions in terms of cell death $(2,3)$. Previous studies have demonstrated that TIPE is an oncogene, and overexpressed TIPE in cells can reduce cell death in vitro and increase tumor growth in vivo $(4,5)$. By contrast, TIPE2, a negative regulator of innate and adaptive immunity, has been demonstrated to be an inhibitor of Ras and to have a pro-apoptotic ability $(2,6,7)$.

However, few studies have been reported about the functions of TIPE1, another member of the TIPE family. Cui et al (8) observed that TIPE1 was expressed in a wide variety of mouse tissues and human carcinoma cell lines, suggesting that TIPE1 may be involved in cell secretion and carcinogenesis. Hitomi et al (9) used a genome small interfering RNA approach to predict that TIPE1 is required for N-benzyloxycarbonyl-Val-Ala-Asp-fluoromethylketone (zVAD, an inhibitor of caspase)- or TNF $\alpha$-induced necrosis in L929 cells, as well as for TNFa/cycloheximide-induced apoptosis in NIH 3 T3 cells. Recently, Zhang et al (10) demonstrated that TIPE1 overexpression in H22 cells could inhibit tumor growth in vivo, and TIPE1 could inhibit the Ras-related C3 botulinum toxin substrate 1 (Rac1)-p65-c-Jun N-terminal kinase (JNK) pathway activation to promote apoptosis in human hepatocellular carcinoma (HCC) cells. Since TIPE1 is expressed in numerous tissues and cell lines, the physiological function and the role in cell death of TIPE1 on the cells of different tissue sources remain to be elucidated.

In present study, a murine macrophage cell line, RAW264.7, was used to investigate the apoptotic function of TIPE1. Our results demonstrated that TIPE1 could promote the apoptosis of RAW264.7 cells by upregulating the pro-apoptotic members of the B-cell leukemia/lymphoma (Bcl)-2 family of proteins, and that sustained activation of the mitogen activated protein 
kinases (MAPKs) signaling pathway was not involved in the process.

\section{Materials and methods}

Reagents. The Annexin V/PI Apoptosis Detection kit was purchased from Nanjing KeyGen Biotech Co., Ltd. (Nanjing, China). Cisplatin [cis-diamminedichloroplatinum II (DDP)] was obtained from Calbiochem (EMD Millipore, Billerica, MA, USA). Dulbecco's modified Eagle medium (DMEM) and fetal bovine serum (FBS) were acquired from HyClone (GE Healthcare Life Sciences, Logan, UT, USA). TaKaRa Taq DNA Polymerase, RNAiso Plus and PrimeScript Reverse Transcriptase were purchased from Takara Biotechnology Co., Ltd. (Dalian, China). Rabbit anti-mouse antibodies against (Bcl)-2 associated X protein (Bax; \#2722; 1:1,000), Bcl-2 interacting killer (Bik; \#4592; 1:1,000), p53 upregulated modulator of apoptosis (Puma; \#7467; 1:1,000), Bcl-extra large (xl; \#2764; 1:3,000), Bcl-2 (\#3498; 1:1,000), phospho-extracellular signal-regulated kinase (Erk) 1/2 (\#4370; 1:1,000), phospho-p38 (\#9212; 1:1,000) and phospho-JNK (\#9251; 1:1,000), as well as U0126 (\#9903), SB203580 (\#5633) and SP600125 (\#8177), were purchased from Cell Signaling Technology, Inc. (Danvers, MA, USA). Rabbit anti-mouse antibody against TIPE1 (\#SAB2102488; 1:1,000) was purchased from Sigma-Aldrich (Merck Millipore, Darmstadt, Germany).

Cell line and culture. RAW264.7 cells with stably transfected MigR1 or MigR1-TIPE1 vector were obtained from Professor Yuhai Chen (University of Pennsylvania School of Medicine, Philadelphia, PA, USA). Cells were cultured in DMEM supplemented with $10 \% \mathrm{FBS}, 100 \mu \mathrm{g} / \mathrm{ml}$ streptomycin and $100 \mathrm{U} / \mathrm{ml}$ penicillin. All cell cultures were maintained at $37^{\circ} \mathrm{C}$ under $95 \%$ relative humidity and $5 \%$ carbon dioxide.

Animals. Athymic mice (female, 6-week-old, 20 g, n=20) were purchased from the Shanghai Laboratory Animal Center of Chinese Academy of Sciences (Shanghai, China) and kept at the Animal Center of Xiamen University (Xiamen, China). Mice were housed in a controlled environment and provided with standard rodent food and water. The present study was approved by the Review Board of Medical College at Xiamen University and was performed in compliance with regulations of Xiamen University on experimental animals.

Analysis of gene expression. Total RNA of cells was isolated using RNAiso Plus. For each sample, $2 \mu \mathrm{g}$ of RNA was subjected to reverse transcription (RT) using PrimeScript Reverse Transcriptase. The complementary DNA was amplified by polymerase chain reaction (PCR) with TaKaRa Taq DNA Polymerase according to the manufacturer's protocol $\left(95^{\circ} \mathrm{C}\right.$ for $5 \mathrm{~min}$ followed by 35 cycles of $95^{\circ} \mathrm{C}$ for $30 \mathrm{sec}, 60^{\circ} \mathrm{C}$ for $30 \mathrm{sec}$ and $72^{\circ} \mathrm{C}$ for $30 \mathrm{sec}$, and $72^{\circ} \mathrm{C}$ for $5 \mathrm{~min}$ ), using a QX200 Droplet Digital PCR system (Bio-Rad Laboratories, Inc., Hercules, CA, USA). The PCR products were analyzed on $2 \%$ agarose gel electrophoresis and visualized with ethidium bromide. $\beta$-actin was used as an internal control. The sequences of the primers used are as follows: $\beta$-actin sense 5'-CATCCG TAAAGACCTCTATGCCAAC-3' and antisense 5'-ATGGAG CCACCGATCCACA-3'; and TIPE1 sense 5'-GCCCTGCAG
GCCCAGAAGAAG-3' and antisense 5'-GCTTCACAGCCA CCTTCACCAGG-3'.

Apoptosis assay. Cell apoptosis assays were conducted as previously reported (11). Briefly, for determining the apoptosis rates of TIPE1-overexpressing cells, 8x10 ${ }^{4}$ RAW264.7 cells were seeded in 24-well plates and treated with or without $1 \mu \mathrm{g} / \mathrm{ml}$ cisplatin (DDP) for $20 \mathrm{~h}$. Next, all cell samples were removed by trypsinization, and the removed cells were rinsed with phosphate-buffered saline (PBS) and stained with annexin $\mathrm{V}$-allophycocyanin and propidium iodide (PI) in binding buffer for $20 \mathrm{~min}$ at room temperature. Then, the cells were washed once with PBS, and flow cytometry was performed with BD FACSCalibur (BD Biosciences, San Jose, CA, USA). Data were analyzed with CellQuest Pro 5.1 software (BD Biosciences).

Tumor growth assay. Tumor implantation experiments were conducted as described previously (12). Briefly, every mouse was challenged with $2 \times 10^{6}$ RAW264.7-TIPE1 or RAW264.7-MigR1 cells through subcutaneous injection in the back. In total, 10 mice were injected with RAW264.7-MigR1 cells (control cells) and 10 mice were injected with RAW264.7-TIPE1 cells (TIPE1-overexpressing cells). The tumor volume was measured every 2 days from day 5 to day 15 . The three major axes ( $a, b$ and $c)$ of the tumors were recorded, and tumor volumes were then calculated using the formula abc/2 as reported (4). The mice were sacrificed on day 15 , when the tumors' longest dimension reached a length of $1.0 \mathrm{~cm}$. Then, tumor weights were recorded.

Western blot analysis. Proteins were extracted in lysis buffer as previously described (11). Briefly, cells were lysed with radioimmunoprecipitation assay buffer, and protein lysates were electrophoresed on $10 \%$ sodium dodecyl sulfate-polyacrylamide gel electrophoresis prior to be transferred to polyvinylidene difluoride (PVDF) membranes (Merck Millipore). The PVDF membranes were incubated with the corresponding primary monoclonal antibodies overnight at $4^{\circ} \mathrm{C}$, followed by incubation with the secondary antibodies for $2 \mathrm{~h}$ at room temperature and detection with enhanced chemiluminescence (Merck Millipore). $\beta$-actin was used as a loading control.

Statistical analysis. Unpaired two-tailed Student's $t$-test was performed with GraphPad Prism 5 (GraphPad Software, Inc., San Diego, CA, USA). $\mathrm{P}<0.05$ was considered to indicate a statistically significant difference.

\section{Results}

TIPE1 overexpression promotes the apoptosis of RAW264.7 cells. Endogenous and exogenous expression of TIPE1 in RAW264.7 cells was verified by RT-PCR. As shown in Fig. 1, RAW264.7 cells could express endogenous TIPE1, and the messenger RNA level of TIPE1 was much higher in TIPE1-overexpressing cells (RAW264.7-TIPE1) than in control vector-expressing cells (RAW264.7-MigR1). Overexpression of TIPE1 was also verified by western blotting (Fig. 1B). Since interference of TIPE1 RNA expression prevents NIH 
A

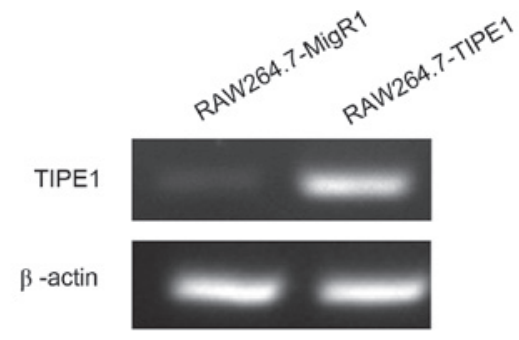

B

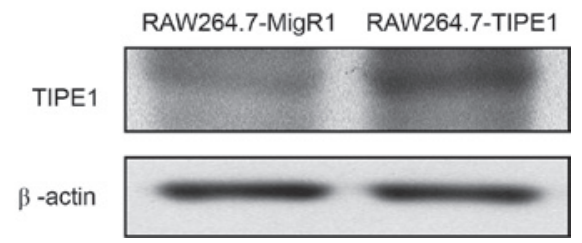

Figure 1. Expression of TIPE1 in RAW264.7 cells. (A) Endogenous and overexpressed TIPE1 in RAW264.7 cells was determined by reverse transcription-polymerase chain reaction. (B) Overexpression of TIPE1 was detected by western blotting. $\beta$-actin was used as a loading control RAW264.7-MigR1, control vector-expressing cells; RAW264.7-TIPE1, TIPE1-overexpressing cells; TIPE1, tumor necrosis factor $\alpha$-induced protein 8-like 1.

$3 \mathrm{~T} 3$ cells (a standard fibroblast cell line) from zVAD-induced apoptosis (9), and TIPE1 overexpression could induce apoptosis of Bel7402 cells (an HCC cell line) (10), the present study investigated whether TIPE1 overexpression could directly affect RAW264.7 cell apoptosis. Flow cytometry analysis with annexin V and PI was used to detect the apoptosis of cells. The results indicated that the apoptosis rates were relatively enhanced by TIPE1, from 5.6 to $11.4 \%$ (Fig. 2, $\mathrm{P}<0.001$ ). Meanwhile, $1 \mu \mathrm{g} / \mathrm{ml}$ cisplatin (DDP), an anti-tumor medicine involving the mitochondrial pathway of apoptosis and causing DNA damage (13), was used to induce cell death in RAW264.7 cells. TIPE1-overexpressing cells also exhibited significantly increased apoptosis rates (38.1\%) compared with control cells (24.0\%) upon DDP stimulation (Fig. 2, P $<0.001$ ). These results suggested that overexpressed TIPE1 could induce apoptosis and enhance the sensitivity of DDP-induced cell death in RAW264.7 cells in vitro.

TIPE1 overexpression suppresses tumor growth in vivo. Since TIPE1 was capable of affecting apoptosis in RAW264.7 cells in vitro, it was hypothesized that the growth of tumors in vivo could be suppressed by TIPE1 overexpression. To test this hypothesis, RAW264.7 cells with or without TIPE1 overexpression were injected subcutaneously into athymic mice. As shown in Fig. 3, TIPE1-overexpressing RAW264.7 cells exhibited a remarkably decreased tumor growth as compared with control cells from day 11 to day 15 . In addition, the tumor weights were also significantly suppressed by TIPE1 overexpression (day 15: RAW264.7-MigR1, $0.20 \pm 0.10 \mathrm{~g}$ vs. RAW264.7-TIPE1, $0.05 \pm 0.02 \mathrm{~g}, \mathrm{P}<0.001)$. These results indicated that TIPE1 could inhibit tumor growth in vivo by inducing apoptosis.

TIPE1 overexpression influences the protein expression levels of the Bcl-2 family to promote apoptosis. Since the intrinsic apoptotic pathway could be initiated by DNA damage $(14,15)$, our in vitro studies (Fig. 2) strongly suggest that TIPE1 promotes apoptosis of RAW264.7 cells by regulating the mitochondrial pathway. It could be assumed that Bcl-2 family proteins, which are important regulators of the mitochondrial pathway of apoptosis, may be involved in the overexpressed TIPE1-induced apoptosis of RAW264.7 cells (16). Bax, Bik and Puma, the pro-apoptotic members of the Bcl-2 family of proteins (16-19), were analyzed by western blotting. Fig. 4A indicates, as expected, that when TIPE1 was overexpressed in RAW264.7 cells, the expression of Bax, Bik and Puma increased distinctly. According to a previous report (10), TIPE1 could inhibit the expression of Bcl-2, an important proliferation regulator of the Bcl-2 family (16), in HCC cells. Considering that $\mathrm{Bcl}-\mathrm{xl}$ is another key regulator of anti-apoptotic members of the Bcl-2 family (20), both Bcl-2 and Bcl-xl levels were analyzed by western blotting. As show in Fig. 4B, contrarily to previous results (10), overexpressed TIPE1 did not influence the level of Bcl-2 in RAW246.7 cells, but slightly enhanced Bcl-xl expression. These results indicated that TIPE1 overexpression could affect the mitochondrial pathway of apoptosis by regulating the pro- and anti-apoptotic proteins of the Bcl-2 family.

TIPE1 overexpression activates the MAPKs signaling pathway. A previous study reported that TIPE1 could inhibit the JNK phosphorylation induced by Rac1 (10). The activation of MAPKs is involved in numerous aspects of the regulation of cellular proliferation and apoptosis $(21,22)$. Therefore, the effects of TIPE1 overexpression on the MAPKs signaling pathway was examined. Western blot analysis revealed that the phosphorylation levels of Erk1/2, p38 and JNK were all enhanced by TIPE1 overexpression (Fig. 5A). To gain insight into the association between the activated MAPKs pathway and upregulated Bcl-2 family proteins, TIPE1-overexpressing RAW264.7 cells were treated with the inhibitor of the Erk1/2 (U0126), p38 (SB203580) or JNK (SP600125) signaling pathway, respectively, and then Bax, Bik, Puma and Bcl-xl levels were assessed by western blotting. As shown in Fig. 5B, unexpectedly, the expression of Bax, Bik and Puma was not decreased but slightly enhanced by U0126, SB203580 and SP600125, respectively. Meanwhile, Bcl-xl expression was also slightly increased by SB203580 and SP600125, while U0126 did not influence its expression. These findings suggested that the sustained MAPKs activation did not contribute to the upregulation of the Bcl-2 family proteins induced by TIPE1 overexpression.

\section{Discussion}

The present study has demonstrated that overexpressed TIPE1 could promote RAW264.7 cell apoptosis in vitro and inhibit tumor growth in vivo. These phenomena are consistent with the fact that TIPE1 overexpression can induce apoptosis and suppress tumor growth in human HCC cells (10). However, compared with HCC cells, TIPE1 overexpression in RAW264.7 cells did not reduce the Bcl-2 levels and had no influence on its expression. At the same time, Bcl-xl was slightly increased by TIPE1. Bcl-xl and Bcl-2 are multi-Bcl-2 homology (BH) domain proteins of the Bcl-2 family (16). Considering that $\mathrm{Bcl}-\mathrm{xl}$ but not $\mathrm{Bcl}-2$ level was changed by TIPE1 overexpression, 


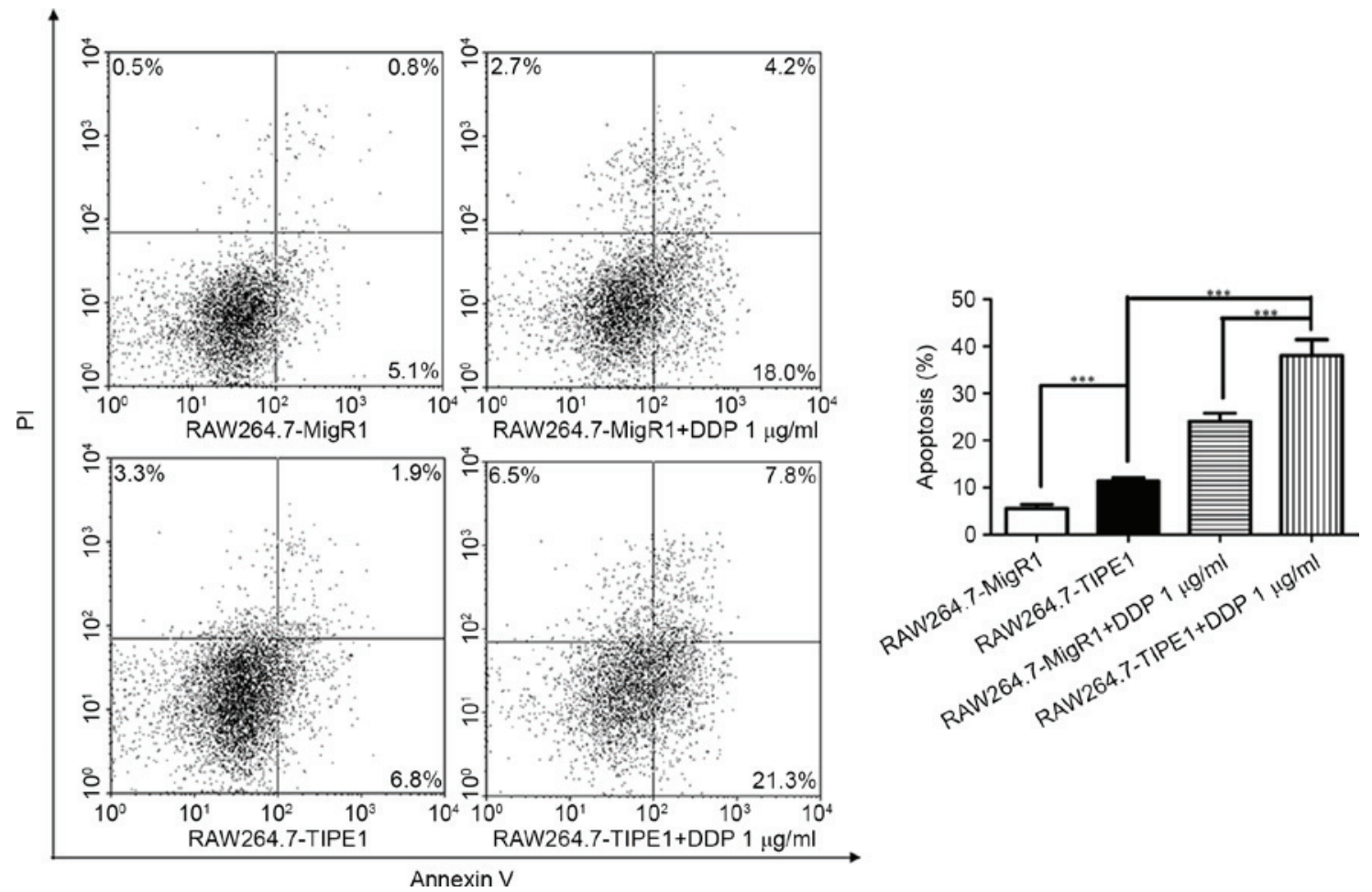

Figure 2. TIPE1 overexpression promotes apoptosis in RAW264.7 cells. RAW264.7 cells were treated with or without $1 \mu \mathrm{g} / \mathrm{ml} \mathrm{DDP}$ for $20 \mathrm{~h}$, and the apoptosis rates were increased by TIPE1 overexpression. The results represent the mean \pm standard deviation of three separate experiments $\left({ }^{* * * *} \mathrm{P}<0.001\right)$. TIPE1, tumor necrosis factor $\alpha$-induced protein 8-like 1; PI, propidium idoide; DDP, cis-diamminedichloroplatinum II.

A

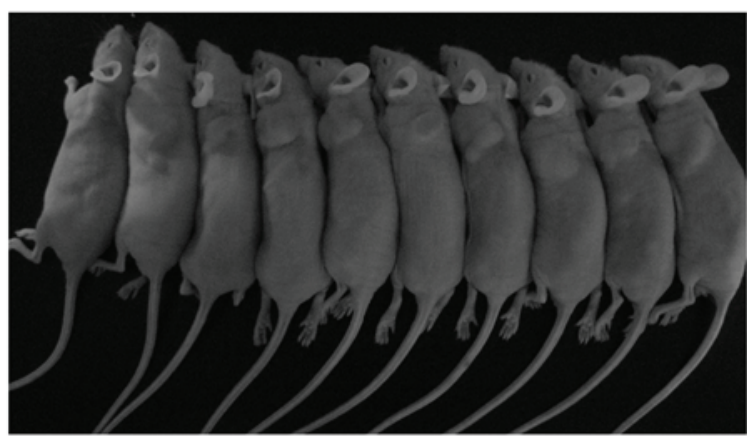

C

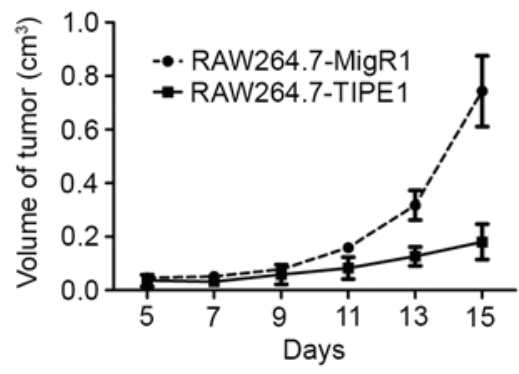

B

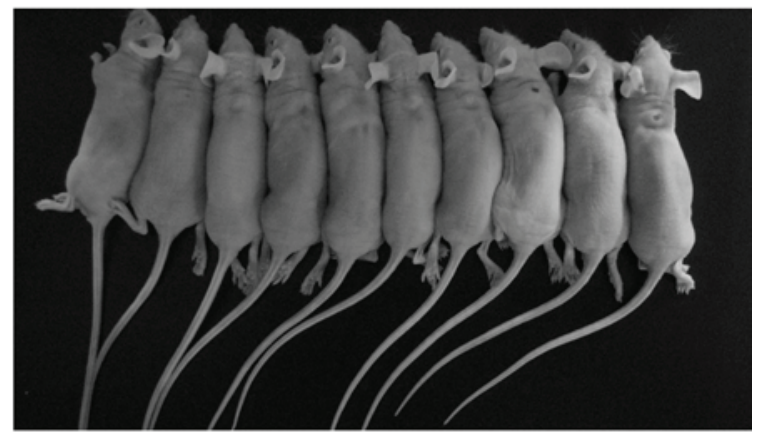

D

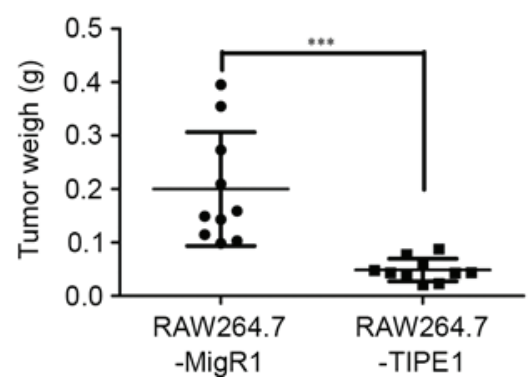

Figure 3. TIPE1 overexpression inhibits tumor growth in vivo. Image of athymic mice with tumors of (A) RAW264.7-MigR1 and (B) RAW264.7-TIPE1. (C) Tumor volumes were examined every 2 days from day 5 after tumor transplantation to day 15 . Data are shown as the mean \pm standard error of the mean. (D) Tumor weights were recorded at day 15 subsequent to athymic mice being sacrificed $\left({ }^{* * *} \mathrm{P}<0.001, \mathrm{n}=10\right)$. Dot represents single tumor weight. Data are shown as the mean \pm standard deviation. TIPE1, tumor necrosis factor $\alpha$-induced protein 8 -like 1 .

Bcl-xl may be the most important anti-apoptotic protein in RAW264.7 cells. On the other hand, it was observed that TIPE1 upregulated pro-apoptotic proteins, including Bax, Bik and Puma, in RAW264.7 cells. These BH-only domain Bcl-2 family proteins, Bik and Puma, could bind and neutralize the pro-survival function of Bcl-xl and Bcl-2 (16,23,24). Compared with Bcl-2, Bik has a higher binding affinity for Bcl-xl, while Puma has nearly similar abilities to bind these 
A

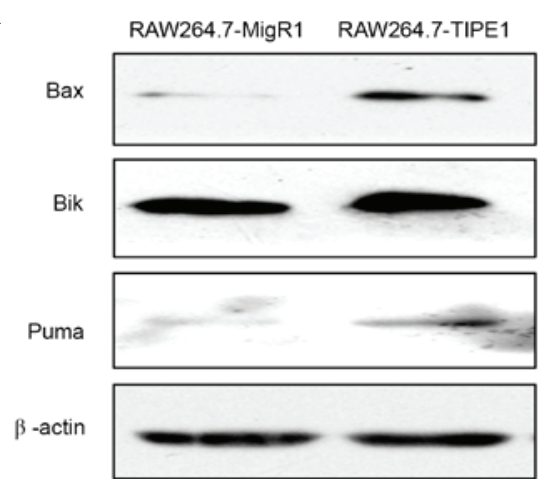

B

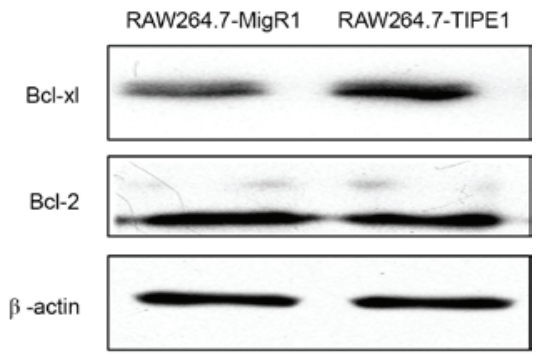

Figure 4. Bcl-2 family proteins expression is influenced by TIPE1 overexpression. The expression of pro- or anti-apoptotic proteins of the Bcl-2 family in RAW264.7 cells was determined by western blotting. (A) TIPE1 could increase the expression levels of Bax, Bik and Puma in RAW264.7 cells. (B) The expression of Bcl-xl in RAW264.7 cells was slightly increased by TIPE1 overexpression, but Bcl-2 expression was not influenced by TIPE1 overexpression. $\beta$-actin was used as a loading control. TIPE1, tumor necrosis factor $\alpha$-induced protein 8-like 1; Bcl-2, B-cell leukemia/lymphoma-2; Bax, Bcl-2 associated X protein; Bik, Bcl-2 interacting killer; Puma, p53 upregulated modulator of apoptosis; Bcl-xl, Bcl-extra large.

$\mathbf{A}$

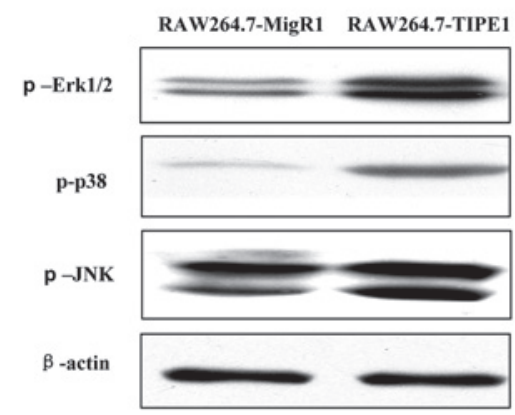

B

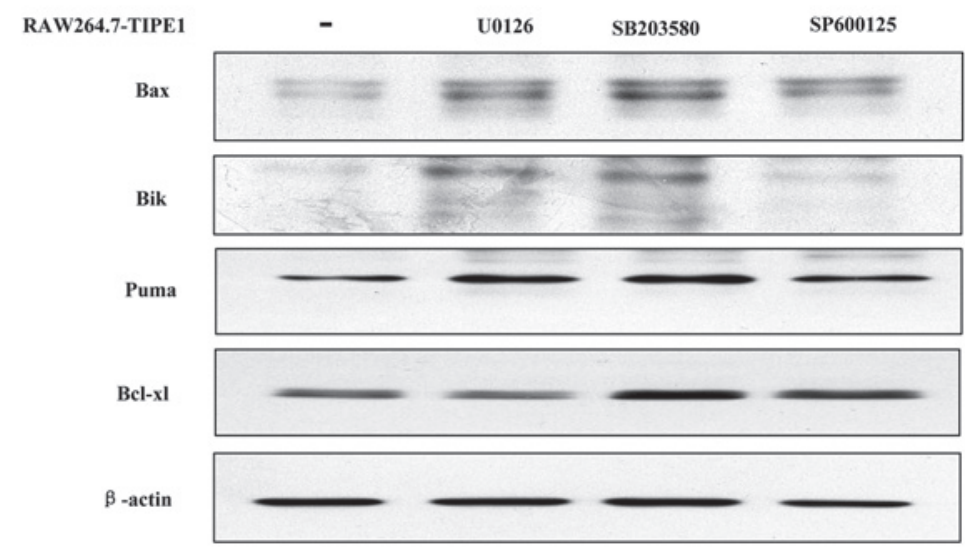

Figure 5. Continuously activated mitogen activated protein kinases signaling pathway did not contribute to the upregulation of the Bcl-2 family of proteins induced by overexpressed TIPE1. (A) Overexpressed TIPE1 enhanced the phosphorylation levels of Erk1/2, p38 and JNK in RAW264.7 cells. (B) RAW264.7-TIPE1 cells were treated with U0126 $(10 \mu \mathrm{M})$, SB203580 $(10 \mu \mathrm{M})$ or SP600125 $(5 \mu \mathrm{M})$ for $15 \mathrm{~h}$, and the levels of Bax, Bik, Puma or Bcl-xl were not reduced by the inhibitors. $\beta$-actin was used as a loading control. TIPE1, tumor necrosis factor $\alpha$-induced protein 8 -like 1; p, phosphorylated; Erk, extracellular signal-regulated kinase; JNK, c-Jun N-terminal kinase; Bax, Bcl-2 associated X protein; Bik, Bcl-2 interacting killer; Puma, p53 upregulated modulator of apoptosis; Bcl-xl, Bcl-extra large; Bcl, B-cell leukemia/lymphoma.

two pro-survival Bcl-2 family proteins (23). In addition, Puma and Bik can also directly activate Bax, a multi-BH domain Bcl-2 family protein and a key pro-apoptotic effector in the mitochondrial pathway to trigger apoptosis $(17,25,26)$. It has been reported that when Bax and Bcl-xl are both upregulated, the cells exhibit a higher apoptosis rate than control cells (17). Thus, we propose that upregulated Bik and Puma could neutralize the pro-survival function of increased Bcl-xl, and that increased Bax could also overcome the anti-apoptotic ability of Bcl-xl while being activated by Bik and Puma to promote apoptosis in TIPE1-overexpressing RAW264.7 cells.

It has been reported that TIPE1 could inhibit the Rac1-induced JNK activation in HEK293 cells (10). Contrarily to this report, the present study revealed that MAPKs, including JNK, were constantly activated by TIPE1 overexpression in RAW264.7 cells. Erk, p38 and JNK could regulate mitochondrial functions, including the levels of Bcl-2 family proteins, to influence apoptosis progression (27-30). However, the present study demonstrated that the expression of pro-apoptotic proteins (Bax, Bik and Puma) could not be reduced by inhibiting Erk1/2, JNK or p38 activation, and the pro-survival factor Bcl-xl was also enhanced. These phenomena indicated that MAPKs activation is not involved in the TIPE1 pro-apoptotic function. Notably, it is known from a previous study that overexpressed TIPE2, another pro-apoptotic member of the TNFAIP8 family, could also constantly activate p38 (31). Besides its pro-apoptotic function, TIPE2 is also an important negative regulator in innate and adaptive immunity, which could influence the expression of several cytokines by negatively regulating JNK and p38 activation in various types of immune cells, including the macrophage cell line RAW264.7 (2,7,32). TIPE, another member of the TNFAIP8 family, has also been reported as a regulator of the inflammatory cytokine interleukin- $1 \beta$ in RAW264.7 cells (33). Similarly, total or partial activation of Erk1/2, JNK and p38 could be observed in immune-related functions in macrophages (including RAW264.7 cells); thus, inhibiting the activation of these MAPKs proteins could 
decrease the expression of various cytokines (34-37). In the present study, TIPE1 was overexpressed in RAW264.7 cells; thus, the phenomenon of sustained MAPKs activation could possibly indicate certain immune-related function of TIPE1.

In conclusion, the present study demonstrated that TIPE1 overexpression could promote apoptosis in RAW264.7 cells and inhibit tumor growth in athymic mice. Overexpressed TIPE1 influenced the intrinsic apoptosis pathway by regulating the expression levels of pro- and anti-apoptotic factors of the Bcl-2 family of proteins, and sustained MAPKs activation did not contribute to the pro-apoptotic progression. Our study supports the idea that TIPE1 is a pro-apoptotic factor. Further studies are required to understand how TIPE1 regulates cell death.

\section{Acknowledgements}

The present study was supported by the Natural Science Foundation of Fujian Province of China (Fuzhou, China; grant no. 2013J05124). We thank Professor Yuhai Chen (University of Pennsylvania School of Medicine, Philadelphia, PA, USA) for kindly providing the RAW264.7 cells with stably transfected MigR1 or MigR1-TIPE1 vector. In addition, we thank Professor Fengguang Gao (Xiamen University, Fujian, China) for his technical advice.

\section{References}

1. Tibbetts MD, Zheng L and Lenardo MJ: The death effector domain protein family: Regulators of cellular homeostasis. Nat Immunol 4: 404-409, 2003.

2. Sun H, Gong S, Carmody RJ, Hilliard A, Li L, Sun J, Kong L, Xu L, Hilliard B, Hu S, et al: TIPE2, a negative regulator of innate and adaptive immunity that maintains immune homeostasis. Cell 133: 415-426, 2008.

3. Kumar D, Whiteside TL and Kasid U: Identification of a novel tumor necrosis factor-alpha-inducible gene, SCC-S2, containing the consensus sequence of a death effector domain of fas-associated death domain-like interleukin-lbeta-converting enzyme-inhibitory protein. J Biol Chem 275: 2973-2978, 2000.

4. Kumar D, Gokhale P, Broustas C, Chakravarty D, Ahmad I and Kasid U: Expression of SCC-S2, an antiapoptotic molecule, correlates with enhanced proliferation and tumorigenicity of MDA-MB 435 cells. Oncogene 23: 612-616, 2004.

5. Miao Z, Zhao T, Wang Z, Xu Y, Song Y, Wu J and Xu H: SCC-S2 is overexpressed in colon cancers and regulates cell proliferation. Tumour Biol 33: 2099-2106, 2012.

6. Gus-Brautbar Y, Johnson D, Zhang L, Sun H, Wang P, Zhang S, Zhang L and Chen YH: The anti-inflammatory TIPE2 is an inhibitor of the oncogenic Ras. Mol Cell 45: 610-618, 2012.

7. Zhang W, Zhang J, Zhao L, Shao J, Cui J, Guo C, Zhu F, Chen YH and Liu S: TIPE2 protein negatively regulates HBV-specific CD8+ T lymphocyte functions in humans. Mol Immunol 64: 204-209, 2015.

8. Cui J, Zhang G, Hao C, Wang Y, Lou Y, Zhang W, Wang J and Liu S: The expression of TIPE1 in murine tissues and human cell lines. Mol Immunol 48: 1548-1555, 2011.

9. Hitomi J, Christofferson DE, Ng A, Yao J, Degterev A, Xavier RJ and Yuan J: Identification of a molecular signaling network that regulates a cellular necrotic cell death pathway. Cell 135: 1311-1323, 2008.

10. Zhang Z, Liang X, Gao L, Ma H, Liu X, Pan Y, Yan W, Shan H, Wang Z, Chen YH and Ma C: TIPE1 induces apoptosis by negatively regulating Rac1 activation in hepatocellular carcinoma cells. Oncogene 34: 2566-2574, 2015.

11. Wang YY,Liu Y, Ni XY, Bai ZH, Chen QY,Zhang Y and Gao FG: Nicotine promotes cell proliferation and induces resistance to cisplatin by $\alpha 7$ nicotinic acetylcholine receptor-mediated activation in Raw264.7 and El4 cells. Oncol Rep 31: 1480-1488, 2014.
12. Gao FG, Wan da F and Gu JR: Ex vivo nicotine stimulation augments the efficacy of therapeutic bone marrow-derived dendritic cell vaccination. Clin Cancer Res 13: 3706-3712, 2007.

13. Siddik ZH: Cisplatin: Mode of cytotoxic action and molecular basis of resistance. Oncogene 22: 7265-7279, 2003.

14. Loewer A, Batchelor E, Gaglia G and Lahav G: Basal dynamics of p53 reveal transcriptionally attenuated pulses in cycling cells. Cell 142: 89-100, 2010

15. Khan KH,Blanco-Codesido M and Molife LR: Cancer therapeutics: Targeting the apoptotic pathway. Crit Rev Oncol Hematol 90: 200-219, 2014.

16. Youle RJ and Strasser A: The BCL-2 protein family: Opposing activities that mediate cell death. Nat Rev Mol Cell Biol 9: 47-59, 2008.

17. Finucane DM, Bossy-Wetzel E, Waterhouse NJ, Cotter TG and Green DR: Bax-induced caspase activation and apoptosis via cytochrome $\mathrm{c}$ release from mitochondria is inhibitable by Bcl-xL. J Biol Chem 274: 2225-2233, 1999.

18. Viedma-Rodriguez R, Baiza-Gutman LA, Garcia-Carrancá A, Moreno-Fierros L, Salamanca-Gómez F and Arenas-Aranda D: Suppression of the death gene BIK is a critical factor for resistance to tamoxifen in MCF-7 breast cancer cells. Int J Oncol 43: 1777-1786, 2013.

19. Nakano K and Vousden KH: PUMA, a novel proapoptotic gene, is induced by $\mathrm{p} 53$. Mol Cell 7: 683-694, 2001.

20. Michels J, Kepp O, Senovilla L, Lissa D, Castedo M, Kroemer G and Galluzzi L: Functions of BCL-X L at the interface between cell death and metabolism. Int J Cell Biol 2013: 705294, 2013.

21. Roux PP and Blenis J: ERK and p38 MAPK-activated protein kinases: A family of protein kinases with diverse biological functions. Microbiol Mol Biol Rev 68: 320-344, 2004.

22. Beug ST, Cheung HH, LaCasse EC and Korneluk RG: Modulation of immune signalling by inhibitors of apoptosis. Trends Immunol 33: 535-545, 2012.

23. Chen L, Willis SN, Wei A, Smith BJ, Fletcher JI, Hinds MG, Colman PM, Day CL, Adams JM and Huang DC: Differential targeting of prosurvival $\mathrm{Bcl}-2$ proteins by their $\mathrm{BH} 3$-only ligands allows complementary apoptotic function. Mol Cell 17: 393-403, 2005.

24. Willis SN, Fletcher JI, Kaufmann T, van Delft MF, Chen L, Czabotar PE, Ierino H, Lee EF, Fairlie WD, Bouillet P, et al: Apoptosis initiated when $\mathrm{BH} 3$ ligands engage multiple $\mathrm{Bcl}-2$ homologs, not Bax or Bak. Science 315: 856-859, 2007.

25. Gillissen B, Essmann F, Graupner V, Stärck L, Radetzki S, Dörken B, Schulze-Osthoff K and Daniel PT: Induction of cell death by the BH3-only Bcl-2 homolog Nbk/Bik is mediated by an entirely Bax-dependent mitochondrial pathway. EMBO J 22: 3580-3590, 2003.

26. Gallenne T, Gautier F, Oliver L, Hervouet E, Noël B, Hickman JA, Geneste O, Cartron PF, Vallette FM, Manon S and Juin P: Bax activation by the $\mathrm{BH} 3$-only protein Puma promotes cell dependence on antiapoptotic Bcl-2 family members. J Cell Biol 185: 279-290, 2009.

27. Sheridan C, Brumatti G, Elgendy M, Brunet M and Martin SJ: An ERK-dependent pathway to Noxa expression regulates apoptosis by platinum-based chemotherapeutic drugs. Oncogene 29: 6428-6441, 2010.

28. El Mchichi B, Hadji A, Vazquez A and Leca G: p38 MAPK and MSK1 mediate caspase- 8 activation in manganese-induced mitochondria-dependent cell death. Cell Death Differ 14: 1826-1836, 2007.

29. Deng Y, Ren X, Yang L, Lin Y and Wu X: A JNK-dependent pathway is required for TNF alpha-induced apoptosis. Cell 115: 61-70,2003.

30. Liu WW, Chen SY, Cheng CH, Cheng HJ and Huang PH: Blm-s, a BH3-only protein enriched in postmitotic immature neurons, is transcriptionally upregulated by p53 during DNA damage. Cell Rep 9: 166-179, 2014.

31. Liu QQ, Zhang FF, Wang F, Qiu JH, Luo CH, Zhu GY and Liu YF: TIPE2 inhibits lung cancer growth attributing to promotion of apoptosis by regulating some apoptotic molecules expression. PLoS One 10: e0126176, 2015.

32. Wang Z, Fayngerts S, Wang P, Sun H, Johnson DS, Ruan Q, Guo W and Chen YH: TIPE2 protein serves as a negative regulator of phagocytosis and oxidative burst during infection. Proc Natl Acad Sci USA 109: 15413-15418, 2012.

33. Ahn SH, Deshmukh H, Johnson N, Cowell LG, Rude TH, Scott WK, Nelson CL, Zaas AK, Marchuk DA, Keum S, et al: Two genes on $\mathrm{A} / \mathrm{J}$ chromosome 18 are associated with susceptibility to Staphylococcus aureus infection by combined microarray and QTL analyses. Plos Pathog 6: e1001088, 2010. 
34. Dai H, Xu D, Su J, Jang J and Chen Y: Transmembrane protein 106a activates mouse peritoneal macrophages via the MAPK and NF- $\kappa$ B signaling pathways. Sci Rep 5: 12461, 2015.

35. Yang CR, Wang JH, Hsieh SL, Wang SM, Hsu TL and Lin WW: Decoy receptor 3 (DcR3) induces osteoclast formation from monocyte/macrophage lineage precursor cells. Cell Death Differ 11 (Suppl 1): S97-S107, 2004.

36. An HZ, Zhao W, Hou J, Zhang Y, Xie Y, Zheng Y, Xu H, Qian C, Zhou J, Yu Y, et al: SHP-2 phosphatase negatively regulates the TRIF adaptor protein-dependent type I interferon and proinflammatory cytokine production. Immunity 25: 919-928, 2006.
37. Xia M, Liu J, Wu X, Liu S, Li G, Han C, Song L, Li Z, Wang Q, Wang J, et al: Histone methyltransferase Ash1l suppresses interleukin- 6 production and inflammatory autoimmune diseases by inducing the ubiquitin-editing enzyme A20. Immunity 39: 470-481, 2013. 